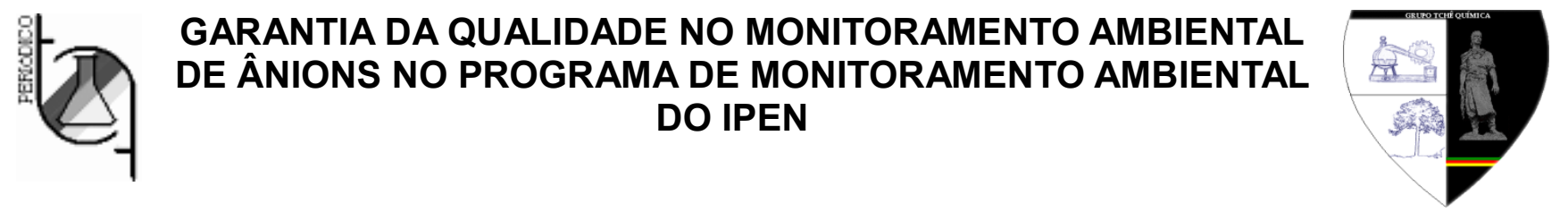

\title{
QUALITY ASSURANCE OF ANIONS ENVIRONMENTAL MONITORING IN IPEN'S ENVIRONMENTAL MONITORING PROGRAM
}

VILLA, Sabrina Moura.*; STELLATO, Thamiris Brandino"; MARQUES, Joyce Rodrigues"; FAUSTINO, Mainara Generoso'; SILVA, Douglas Batista'; MONTEIRO, Lucilena Rebelo ${ }^{1}$, DA SILVA, Tatiane B. S. Carvalho'; COTRIM, Marycel E. Barbosa'; PIRES, Maria Aparecida F'.

${ }^{1}$ Instituto de Pesquisas Energéticas e Nucleares /Comissão Nacional de Energia Nuclear, Av. Lineu Prestes, 2242, São Paulo- Brasil,

* Autor correspondente

e-mai: sabrinamvilla@outlook.com

Received 17 November 2016; received in revised form 24 November 2016; accepted 08 December 2016

\section{RESUMO}

Este trabalho visa avaliar o controle de qualidade interno e externo da analise de ânions, realizado no IPEN, utilizando a técnica de cromatografia de íons e métodos estatísticos para a análise de dados. Portanto foi possível concluir que o sistema esta sob controle, gerando resultados confiáveis

Palavras-chave: Controle de Qualidade interno, Controle de Qualidade Externo, Cromatografia de Íons

\section{ABSTRACT}

This work aims to assess the internal and external quality control of the anion analysis, accomplished at IPEN, using chromatography technique ions and Statistical Methods for data analysis. So it was possible to conclude that the system is over control, generating reliable results

Keywords: Internal Quality Control, External Quality Control, Ion Chromatography

\section{INTRODUÇÃO}

O Programa de Monitoramento Ambiental de compostos químicos estáveis- PMA-Q, do Instituto de Pesquisas Energéticas e nucleares IPEN avalia entre outros parâmetros os ânions em água subterrânea e efluentes, da rede coletora de esgoto, utilizando o método de cromatografia de íons (IPEN, 2011)

A implantação de mecanismos de Controle de Qualidade nos assegura quanto à eficiência do laboratório. Esses controles possibilitam a documentação do comportamento de um determinado sistema, em um laboratório específico, familiarizam seu operador com as condições de rotina, e o capacitam a avaliar corretamente o resultado.

No Estado de São Paulo, para o monitoramento ambiental, são necessárias ações de Controle de Qualidade ou o Sistema da Garantia da Qualidade, desde a amostragem até a emissão de Relatórios de análise (Estado de São Paulo, 2012), sem esse controle, não é possível ter uma exatidão da veracidade de um determinado resultado podendo este ser aproximado ou até mesmo incorreto.

Um Sistema de Garantia da Qualidade descreve e documenta procedimentos, equipamentos e checagens analíticas a fim de assegurar que um laboratório atinja um 
determinado padrão de qualidade e que este seja monitorado e mantido continuamente, obtendo assim a confiabilidade do resultado. Portanto os critérios de reconhecimento e correção de baixo desempenho, quanto as atribuições de quem deve tomar as ações corretivas, devem ser identificados e estabelecidos pelo laboratório.

O Centro de Química e Meio Ambiente CQMA conta com o Sistema da Qualidade implantado desde 1997. Neste relatório são descritas as ações de Controle de Qualidade internas e externas referente aos ensaios de Cromatografia de Íons nos três últimos anos visando o atendimento a Norma ISO 17025 e em atendimento a melhorias exigidas na Resolução da Secretaria de Meio Ambiente de São Paulo SMA90/2012 (Estado de São Paulo, 2012) e nas resoluções do CONAMA 430/2011 (Brasil, 2011) e 396/2008 (Brasil, 2008).

\section{MATERIAL E MÉTODOS}

Todos os ensaios de ânions foram realizados com o Cromatógrafo de ĺons, marca DIONEX modelo DX120, equipado com sistema supressor auto regenerante, detector de condutividade e injetor com loop de $100 \mu \mathrm{L}$, e o Software Chromeleon 6.8, de acordo com a metodologia APHA 4110 (APHA AWWA WPCF, 2005).

As curvas analíticas são preparadas conforme a Tabela 1. Estas soluções são preparadas empregando padrão de Sete ânions (Thermo Fisher, USA) rastreável ao NIST. Antes dos ensaios, o sistema é estabilizado por 15 minutos e um branco e um padrão de verificação da curva são analisados. Somente após os valores de branco serem inferiores ao Limite de Quantificação, e quando o padrão de verificação é quantificado com $\pm 10 \%$ de desvio padrão relativo é que as amostras são analisadas.

Tabela 1: Faixa de concentração, em mg. $\mathrm{L}^{-1}$ empregados na curva analítica.

\begin{tabular}{l|c|c|c|c|c}
\hline Ânion & PD1 & PD2 & PD3 & PD4 & PD5 \\
\hline$F^{-}$ & 0,1 & 0,2 & 0,4 & 2 & 4 \\
$\mathrm{C}^{-}$ & 0,5 & 1 & 2 & 10 & 20 \\
$\mathrm{NO}_{3}$ & 0,5 & 1 & 2 & 10 & 20 \\
$\mathrm{NO}_{-2}$ & 0,5 & 1 & 2 & 10 & 20 \\
$\mathrm{SO}_{4}^{-2}$ & 0,5 & 1 & 2 & 10 & 20
\end{tabular}

\subsection{Cartas controle de qualidade interno}

A solução denominada PD3 correspondente ao ponto intermediário da curva analítica que é preparada por diluição do Padrão 7 Anion Standard (Thermo Fisher, USA). As concentrações nominais dos ânions, correspondentes as concentrações de preparo, são apresentadas na Tabela 2. Esta solução foi medida 40 vezes em ensaios independentes realizadas entre 14/03/2012 e 01/11/2013. Com o resultado desses ensaios foi calculada a média e o desvio padrão $(\sigma)$ para cada ânion. As cartas controles foram construídas conforme o recomendado no item 9.2 da Norma ISO 13528 (ISO13528, 2005). Os limites de operação foram estabelecidos pela média $\pm 1 \sigma$, sendo estes valores de operação normal, valores observados acima da média $\pm 2 \sigma$ foram considerados valores de alerta e um único valor se medido acima da média $\pm 3 \sigma$ é considerado como fora do limite de operação normal, requerendo, portanto, ações corretivas. Estas ações também foram programadas no caso da ocorrência de dois ou três valores de alerta em sequência.

\subsection{Cartas controle de qualidade externo}

Para o controle de qualidade externo, foram empregados os resultados de Escore- Z obtidos pelo laboratório desde 2011, no Programa de Ensaios de Proficiência em Cromatografia lônica da Rede Metrológica do Rio Grande do Sul (Rede Metrológica do Rio Grande do Sul, 2010).

A participação em programas interlaboratoriais e a avaliação através do Escore-Z permitem:

- Determinar o desempenho individual dos laboratórios para os ensaios propostos;

- Monitorar de forma contínua o desempenho dos laboratórios;

- Identificar tendências positivas ou negativas (Bias) nos processos de medição;

- $\quad$ Propiciar subsídios aos laboratórios para a identificação e solução de problemas analíticos;

- Identificar diferenças interlaboratoriais;

Os resultados dos ensaios foram avaliados de acordo com o Escore-Z, calculado conforme a Norma ISO 13528 (ISO13528, 2005), 
que pode ser encontrado no relatório anual de 2012 no Anexo B, para os quais foram adotados os seguintes critérios de desempenhos dos participantes:

São classificados como SATISFATÓRIO, QUESTIONÁVEL ou INSATISFATÓRIO, para cada um dos parâmetros em análise, conforme o valor do Escore Z:

Se: $\begin{array}{ll} & |Z| \leq 2 \quad \text { Resultado Satisfatório } \\ & 2<|Z|<3 \text { Resultado Questionável } \\ & |Z| \geq 3 \quad \text { Resultado Insatisfatório. }\end{array}$

Ações corretivas foram planejadas para o caso de ocorrerem resultados Questionáveis ou Insatisfatórios, com o objetivo de corrigir esses desvios no desempenho do laboratório. As tendências, caracterizadas por mais de três resultados de Escore- $Z$ positivos $(Z>0)$ ou negativos $(Z<0)$ também são discutidas abaixo.

\section{RESULTADOS E DISCUSSÃO:}

\subsection{Controle de qualidade Interno}

A média das concentrações dos ânions, os desvios padrão e os desvios padrão relativos (RSD) observados no período de 2 anos, em 40 medidas independentes também são mostrados na Tabela 2. A diferença observada entre a concentração nominal e a média das concentrações medidas para cada um dos ânions foi denominada de Tendência (Bias).

As médias das concentrações de Fluoreto, Cloreto, Nitrito-N, Nitrato-N e Sulfato concordaram com as concentrações nominais correspondentes a concentração de preparo. As diferenças entre a concentração média e a de preparo (Tendência) variaram entre $-2,69 \%$ para o Cloreto e 1,59\% para o Sulfato. O desvio padrão relativo $(n=40)$ no período de 2 anos foi considerado aceitável, variando entre $3 \%$ para o Nitrito-N até um valor máximo de $8 \%$ para o Fluoreto. Estes valores são considerados satisfatórios para o intervalo de concentração avaliado.

Em 2013 ocorreram valores fora da condição normal de operação por conta de uma contaminação na coluna de guarda do equipamento pelo adsorvedor do tipo zeólita no mês de Junho. Quando identificada a ocorrência desses valores fora da condição normal de operação, em todos os ânions citados, foi feita ações corretivas que consistiram na limpeza do sistema, na troca de filtros de coluna, troca de coluna de guarda e recalibração do sistema.

Para o Fluoreto e Cloreto os valores fora das condições de operação ocorreram no mês de Maio de 2013, foi julgado como erro de preparo, pois houve uma replicata de valores altos nos demais ânions. Após a preparação de um novo padrão, ações corretivas foram tomadas para que a operação voltasse às condições normais.

Para Nitrito-N e Nitrato-N no ano de 2012, foram observados valores fora da condição de operações decorrentes da degradação das soluções, pois estas espécies são nutrientes consumidos no crescimento de micro-organismos e no caso do Nitrito-N se oxidam convertendo-se a Nitrato-N. Com o preparo de soluções recentes e a repetição das medidas o sistema retornou aos limites de operação normal.

Valores medidos na faixa correspondente de alerta foram observados individualmente, retornando em seguida a valores de operação normal, para todos os ânions. Ou seja, não foram observados dois ou mais valores de alerta seguidos, o que também caracteriza a operação fora de controle pela Norma ISO13528 (ISO13528, 2005) observado em ocasiões distintas. Neste caso não foram realizadas ações corretivas.

Com a média aritmética dos valores de 2012 a 2013 no controle de qualidade interno o sistema em nenhum momento se entrou fora do limite de operação, como observado na Figura 1.

\subsection{Controle de Qualidade Externo}

Os controles de qualidade externos correspondentes a participação em programas de ensaios de proficiência e avaliados através do Escore $-Z$ de cada um dos ânions, no período de 2 anos, são mostrados na Figura 2 a baixo.

Tanto em 2012 quanto em 2013 foram observados $100 \%$ de resultados considerados satisfatórios, na participação dos programas interlaboratoriais específicos para a Cromatografia de íons.

O acompanhamento dos sucessivos valores do Escore Z, para os ânions permite 
identificar o posicionamento relativo do desempenho do laboratório em comparação ao programa de proficiência. Desta forma, valores sucessivos de Escore- $Z$ positivos $(Z>0)$ pode caracterizar uma tendência a superestimar valores de um ânion, ou Escore- $Z$ negativos $(Z<0)$, podem indicar valores subquantificados.

Considerando este critério, nenhum dos ânions analisados apresentou todos os valores de Escore-Z sempre positivos ou sempre negativos, ainda que os resultados fossem satisfatórios em todas as medidas, sem demonstrar valores sistemáticos acima ou abaixo da média de consenso.

\subsection{Adequação à legislação ambiental}

A Tabela 2 mostra uma comparação entre a faixa de trabalho estabelecida para a medida de ânions por cromatografia de íons no efluente lançado pelo IPEN em rede coletora de esgoto e em água subterrânea e as legislações ambientais vigentes no Brasil e em particular no estado de São Paulo. Estas legislações foram adotadas por que a Resolução n420/2009 (9) do CONAMA estabelece valores orientadores e condições de monitoramento para águas subterrâneas, a Resolução $n^{\circ} 430 / 2011$ (3) do CONAMA dispõe de parâmetros e condições para lançamento em efluentes em rede coletora de esgoto; a Portaria 2914/2011 (10) do Ministério da Saúde estabelece normas e padrão para potabilidade de água; e o Decreto Estadual 8486/76 (11) do estado de São Paulo, dispõe sobre a prevenção e o Controle da Poluição do Meio Ambiente, também estabelecendo valores máximos permitidos para o lançamento em rede coletora de esgoto.

Considerando a comparação entre a faixa de trabalho medida e os limites legais estabelecidos nas legislações estaduais e federais, avaliou-se que as medidas de ânions realizadas no IPEN são adequadas para atividades de monitoramento ambiental.

São monitoradas no IPEN concentrações da ordem das concentrações estabelecidas em legislação ou inferiores. Para as concentrações legisladas que excedem a faixa de trabalho, as amostras quando necessário são diluídas e adequadas a faixa de trabalho. Entretanto, em geral, os valores medidos tanto no efluente quanto nas águas subterrâneas, encontram-se dentro da faixa de trabalho e portanto abaixo dos valores máximos estabelecidos na legislação.

Portanto as medições são feitas dentro das condições limite estabelecidas como de operação normal, atendem as faixas de valores exigidos pelas legislações ambientais vigentes, referentes ao lançamento de efluentes e ao monitoramento de água subterrânea.

\section{CONCLUSÕES:}

Pode-se verificar que o controle de qualidade interno e externo referente aos ensaios de ânions em água subterrânea e efluentes da rede coletora de esgoto, durante os dois últimos anos (2012 e 2013) atendem aos requisitos das normas ISO 17025 e ISO 13528 e com o controle de qualidade interno foi possível comprovar também que os ensaios realizados neste período estiveram em condições de operação controlada, sem variação significativa nas condições de ensaio. Com o controle de qualidade externo, foi possível atestar que os resultados emitidos durante o período avaliado, são comparáveis à média de consenso realizada por outros laboratórios participantes do programa interlaboratorial em que empregam a mesma técnica de ensaio. Também se pode concluir que faixa de trabalho empregada atende a faixa de concentração exigida pela legislação ambiental vigente.

\section{REFERÊNCIAS:}

1. IPEN. RELATÓRIO DE AVALIAÇÃO DO PROGRAMA DE MONITORAÇÃO AMBIENTAL(PMA-Q) ATENDENDO AOS COMPOSTOS QUÍMICOS ESTÁVEIS DO IPEN - 2011. São Paulo: Institucional Ipen/CNEN, 2011.

2. Estado de São Paulo. RESOLUÇÃO SMA N ${ }^{\circ} 90$, . [Online] 13 de Novembro de 2012. [Citado em: 27 de Fevereiro de 2013.] http://www.ambiente.sp.gov.br/legislac ao/resolucoes-sma/resolucao-sma-no- 
90-de-13-de-novembro-de-2012/.

3. Brasil. Resolução CONAMA 430. Ministério do Meio Ambiente. [Online] 13 de Maio de 2011. [Citado em: 12 de 12 de 2011.] http://www.mma.gov.br/port/conama/le giabre.cfm?codlegi=646.

4. - Resolução CONAMA No. 396/08. 07 de Abril de 2008.

5. APHA AWWA WPCF. American Public Health Association American Water Works Association \& Water Pollution Control Federation- Standard Methods for the examination of water and wastewater, 21a. edição. Washington, DC(USA) : s.n., 2005.

6. ISO13528. 2005.

7. Rede Metrológica do Rio Grande do Sul.

http://www.redemetrologica.com.br/20 10/. Rede Metrológica do Rio Grande do Sul. [Online] 2010. [Citado em: 16 de Maio de 2012.] http://www.redemetrologica.com.br/20 $10 \%$.

8. Brasil. Resolução CONAMA 420. Ministério do Meio Ambiente. [Online] 30 de dezembro de 2009. [Citado em: 12 de fevereiro de 2013.] http://www.mma.gov.br/port/conama/le giabre.cfm?codlegi=620.

9. Brasil, Ministério da Saúde. Portaria 2914. Brasília : s.n., 12/12/2011.

10. Estado de São Paulo. DECRETO No 8.468, de 08 DE SETEMBRO DE 1976. [Online] 08 de Setembro de 1976. [Citado em: 08 de Julho de 2011.]

http://www.cetesb.sp.gov.br/Institucion al/documentos/Dec8468.pdf.

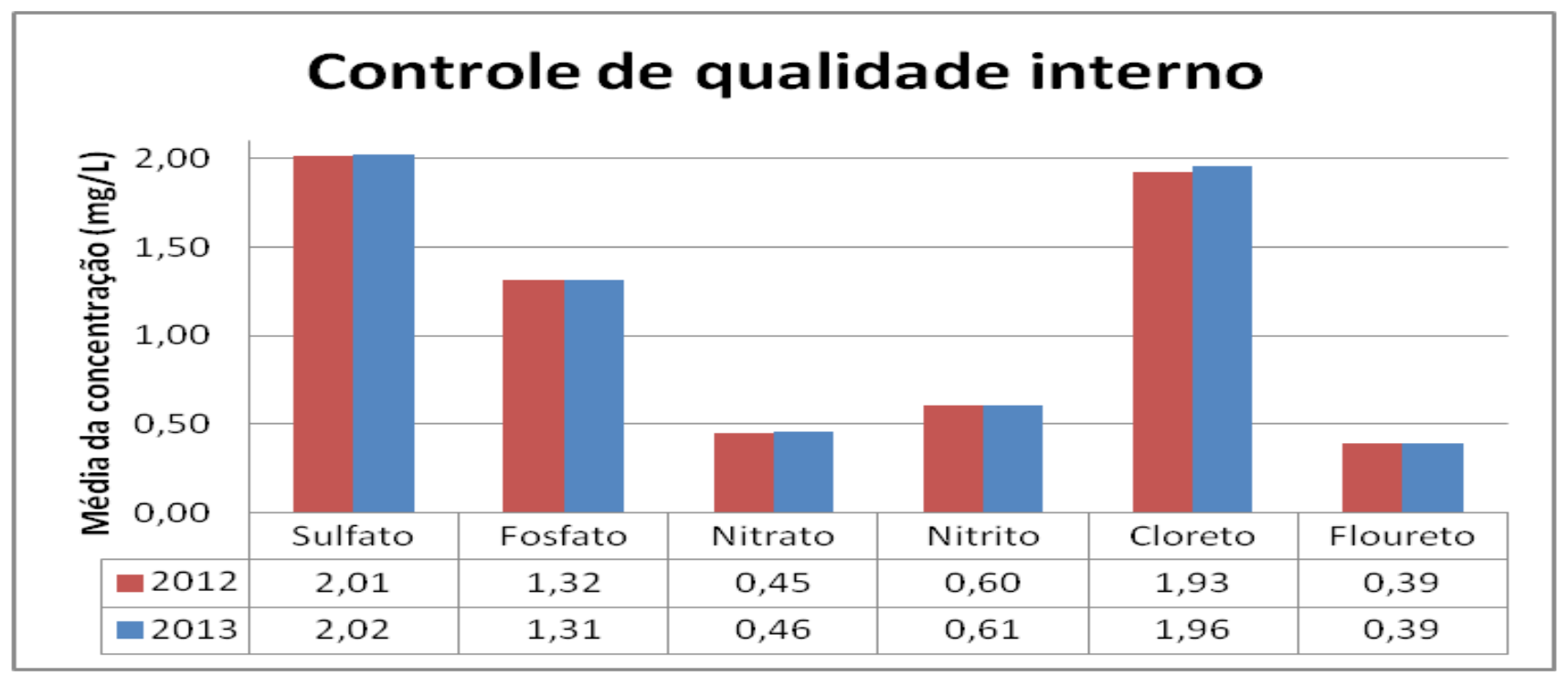

Figura 1: Cartas controle de Qualidade Interno para Fluoreto, Cloreto, Nitrito-N, Nitrato-N, Sulfato e Fosfato, observados pelo período de 2 anos. 


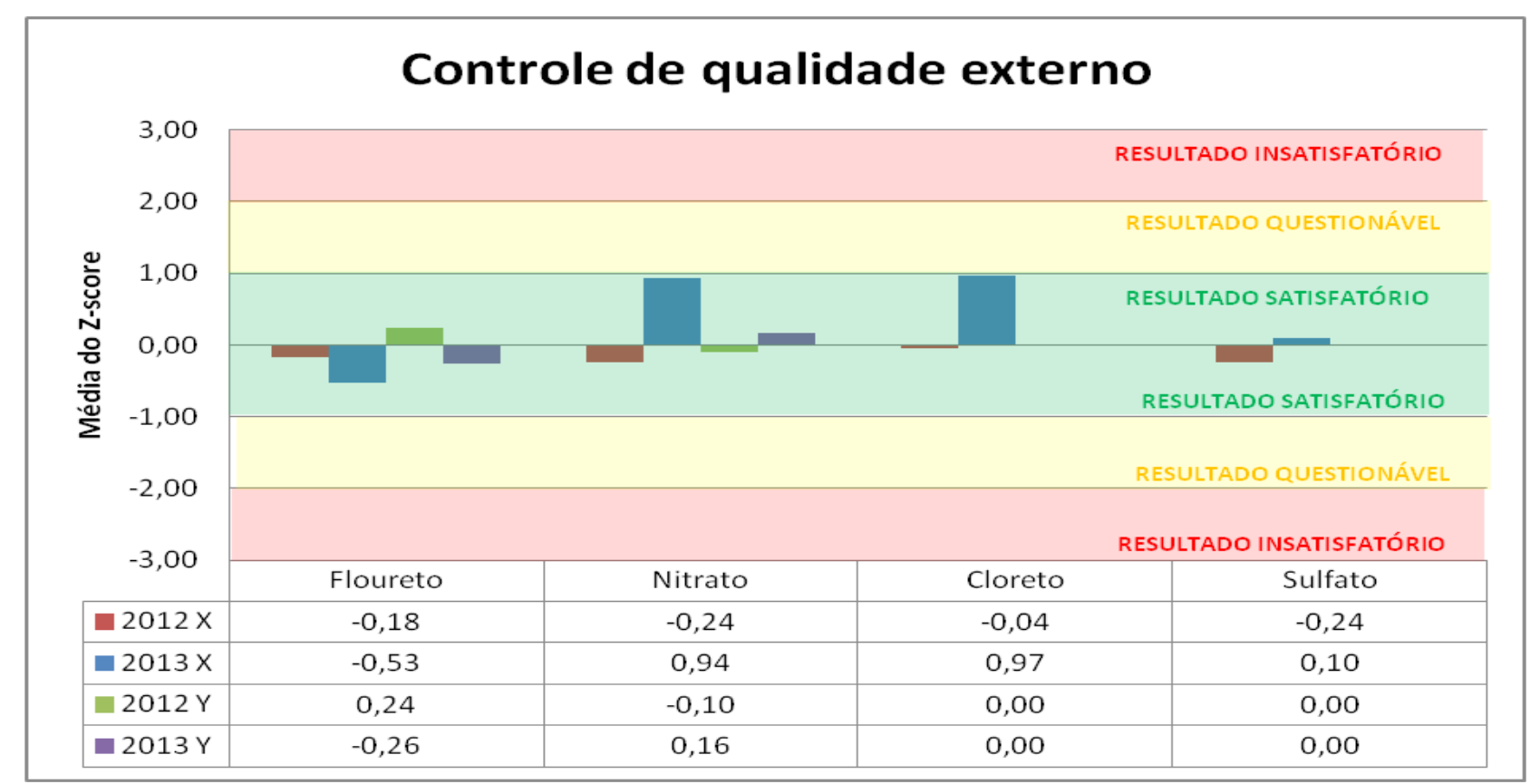

Figura 2: Controle de Qualidade Externo realizado através da avaliação do Escore-Z em Programas Interlaboratoriais para Fluoreto, Cloreto, Nitrato-N e Sulfato, observados pelo período de 2 anos.

Tabela 3:Comparação entre a faixa de trabalho e as legislações ambientais vigentes.

\begin{tabular}{cccccc}
\hline Parâmetro & $\begin{array}{c}\text { Faixa de trabalho } \\
\left(\mathrm{mg} \cdot \mathrm{L}^{-1}\right)\end{array}$ & $\begin{array}{c}\text { Resoluçãon } \\
\left(\mathrm{mg} \cdot \mathrm{L}^{-1}\right)\end{array}$ & $\begin{array}{c}\text { Resoluçãon }{ }^{\circ} 430 \\
\left(\mathrm{mg} \cdot \mathrm{L}^{-1}\right)\end{array}$ & $\begin{array}{c}\text { Portaria } \\
2914 \\
\left(\mathrm{mg} \cdot \mathrm{L}^{-1}\right)\end{array}$ & $\begin{array}{c}\text { Decreto Estadual } \\
8468 \\
\left(\mathrm{mg} \cdot \mathrm{L}^{-1}\right)\end{array}$ \\
\hline $\mathrm{F}$ & $0,02-2,00$ & - & 10 & 1,5 & 10 \\
$\mathrm{Cl}$ & $0,1-20,00$ & - & - & 250 & - \\
$\mathrm{NO}_{3}-\mathrm{N}$ & $0,03-3,04$ & 10 & - & 10 & 10 \\
$\mathrm{NO}_{2}-\mathrm{N}$ & $0,02-4,50$ & - & - & 1,0 & 1,0 \\
$\mathrm{SO}_{4}$ & $0,1-20,00$ & - & - & 250 & 1000 \\
\hline
\end{tabular}

PERIÓDICO TCHÊ QUÍMICA • www.periodico.tchequimica.com • Vol. 14 N. 27.

• ISSN 1806-0374 (impresso) • ISSN 1806-9827 (CD-ROM) • ISSN 2179-0302 (meio eletrônico)

(C) 2017. Porto Alegre, RS. Brasil

The Periódico Tchê Química (ISSN: 1806-0374; 2179-0302) is an open-access journal since 2004. Journal DOI: 10.52571/PTQ. http://www.tchequimica.com.

This text was introduced in this file in 2021 for compliance reasons.

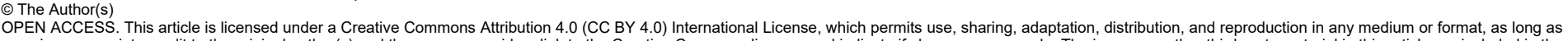
you give appropriate credit to the original author(s) and the source, provide a link to the Creative Commons license, and indicate if changes were made. The images or other third-party material in this article are included in the article 's Creative Commons license unless indicated otherwise in a credit line to the material. If material is not included in the article's Creative Commons license and your intended use is not permitted by statutory regulation or exceeds the permitted use, you will need to obtain permission directly from the copyright holder. To view a copy of this license, visit http://creativecommons.org/licenses/by/4.0/. 